A $R$ R T T I I C C U L L O O $\begin{array}{lllllllll}R & E & F & L & E & X & I & O & N\end{array}$

\title{
FAMILIAS DIVERSAS, DEMOCRACIA Y Diversidad. Presencia y ocultamiento EN LA CIUdAd de Cartagena
}

\author{
DIVERSE FAMILIES, DEMOCRACY AND DIVERSITY. PRESENCE AND \\ HIDING IN THE CITY OF CARTAGENA
}

Por: Lizett López Bajo*

${ }^{*}$ Candidata a magiste en Ciencias Políticas por la Facultad Latinoamericana de Ciencias Sociales, sede Ecuador. Sociales, sede Ecuador. Filósofa de la Universidad de Cartagena, Integrante del Semillero: Familias, feminidades y masculinidades, adscrito a la Facultad de Ciencias Sociales de la Universidad de Cartagena.

Recibido: 15 de marzo de 2017 - Aprobado: 8 de mayo de 2017

\section{RESUMEN}

En este trabajo proponemos hacer una reconceptualización de como se ha entendido y problematizado el concepto de familia. Partimos del hecho que este concepto no es monolítico y presenta distintos matices que deben ser tenidos en cuenta a la hora de estudiar el tema de su conformación y funcionalidad. Se parte de un enfoque de género desde el cual se aborda la temática de la familia en Colombia. Para ello, damos cuenta del contexto dentro del cual surge como una problemática. Incluimos en nuestra propuesta teórica una triada que será el eje de análisis: género-diversidaddemocracia con el fin de contextualizar la inclusión de las familias diversas en el desarrollo de las políticas de inclusión como vía a la democratización. Se quiere mostrar cuales son las dificultades que atraviesan estas familias y la razón de ser del ocultamiento en el que se encuentran. La pregunta central está orientada a dar pistas acerca de qué forma una sociedad verdaderamente democrática incluye en sus legislaciones y acoge en sus modos vivendis la diversidad y la pluralidad con respeto a la diferencia.

Palabras clave: familia, diversidad, democracia, Caribe colombiano.

\begin{abstract}
In this paper a reconceptualization of how the concept of family has been understood and problematized it is proposed. It is started from the fact that this concept is not monolithic and presents different nuances that must be taken into account when studying the theme of its conformation and functionality. It is based on a gender approach from which the issue of family in Colombia is addressed. To do this, the context within which emerges as a problematic is analized. In the theoretical proposal a triad that will be the axis of analysis it is included: gender-diversitydemocracy in order to contextualize the inclusion of diverse families in the development of inclusion policies as a way to democratization. It wants to show what are the difficulties that these families are going through and the reason for the concealment in which they are. The central question is aimed at giving clues as to how a truly democratic society includes in its legislations and receives in its living modes diversity and plurality with respect to difference.
\end{abstract}

Keywords: family, diversity, democracy, colombian caribbean. 


\section{Introducción}

Tr os estudios sobre la familia son fundamentales en las ciencias sociales y en la construcción de la historia social. La familia, como sujeto histórico les también un hecho demográfico, económico, jurídico y esencialmente el conjunto de vínculos humanos, es decir, culturales, funciona como "una organización micro-social que se alberga bajo un techo, comparte el fuego y planifica las trayectorias de vida de sus integrantes, parientes o no, dentro de su entorno social" (Cicerchia, 2012, pág. 11). Hablar de las familias, es hablar de la diversidad de sus formas de constitución, los modos en los que se relacionan los integrantes de la familia, la manera cómo viven y las formas en que se desarrollan los rituales cotidianos como bien ha señalado Bárbara Zapata (2009) en su trabajo sobre las familias.

La indagación y el objeto particular de estudio de este trabajo, no es neutral, no se trata solo de reconocer diversidades y complejidades al interior de la familia. El objetivo central, apunta a mostrar los procesos y las tendencias que ayudan a la democratización, en el interior de las familias como en la relación entre la variedad de formas de familia. Es por ello que investigar sobre la homoparentalidad en el Caribe colombiano es un compromiso con la academia y con las ciencias sociales. En el caso colombiano, el sentido de lo que significa ser familia se viene ampliando y las luchas de actores sociales en la defensa por su derecho a serlo con la puesta en marcha de acciones constitucionales que abrieron la posibilidad a un debate por parte de la ciudadanía sobre la conformación de familia en Colombia y la posibilidad que parejas homoparentales puedan adoptar, se replantea y cuestiona los postulados tradicionales sobre la familia.

En primer lugar, damos cuenta del contexto dentro del cual surge como una problemática. Incluimos en nuestra propuesta teórica una tríada que será el eje de análisis: género-diversidad-democracia con el fin de contextualizar la inclusión de las familias homoparentales en el desarrollo de las políticas de inclusión como vía a la democratización. El punto de partida es la sentencia C-511 de 2011, la cual señala la posibilidad de celebrar una unión matrimonial de las parejas del mismo sexo, hasta la consecución de la adopción y finalmente el matrimonio en el 2016. Con este fin, pasando a una segunda parte, la metodología toma algunos datos cuantitativos de la conformación de familias homoparentales y la elaboración de entrevistas a activistas sociales que han trabajados por este tema en la ciudad de Cartagena y el Caribe, con los problemas y retos a los que se enfrentan. 
La tercera parte de este trabajo presenta los resultados encontrados a partir de las entrevistas y los datos cuantitativos dan cuenta de los avances y retrocesos, así como de la política del Distrito con el fin de hacer posible la inclusión de familias homoparanteles. Los resultados están orientados a la elaboración de posibles alternativas frente a los problemas y déficits de inclusión en medio del binomio que se presenta como imperante en esta situación que es el de: diversidad/tradición. Finalmente, algunas conclusiones sobre lo que hace falta por hacer en este campo tanto a nivel de acciones concretas como a nivel teórico.

\section{Los estudios de género frente al debate sobre las familias}

T a democracia, la entendemos como la idea de la actividad colectiva y participativa de los ciudadanos en la esfera pública. Si como Whitehead لـ (2011) partimos de la tesis de que el proceso de democratización es lento, complejo y parcial, entonces la "metáfora teatral" debe complementarse con un relato más amplio. De ser así, antes que una transición hacia la democracia debe existir una comunidad política que sea receptiva a los ideales y valores democráticos. De ahí que con la democracia se susciten tantos debates, pues es a su vez promesa y problema (Rosanvallon, 2006). Como promesa, la democracia se manifiesta como un régimen acorde a las necesidades ciudadanas y se funda sobre la realización de un doble imperativo de igualdad y autonomía. El problema se presenta en la realidad cuando tales imperativos no logran conjugarse o no se cumplen y como consecuencia de esta situación, el descontento y el hartazgo ciudadano se hacen presentes.

Dentro de este espectro de promesas inconclusas se podría pensar que los sistemas democráticos han sido defectuosos, esto es cierto, pero este sistema ha brindado las herramientas discursivas y de acción para que los ciudadanos construyan redes de ayuda. Logró por ejemplo que temas que se reservaban al espacio privado se hicieran públicos, de ahí, la especial protección a la familia en la constituciones políticas, la cual, deja entonces muchas preguntas sin una respuesta clara aún.

En primer lugar ¿̇a qué tipo de familia es a quien se le otorga especial protección? Segundo ¿bajo qué condiciones son consideradas las familias sujetos especiales de protección? Desde la promulgación de la nueva Constitución en Colombia, la evolución de la definición de familia ha avanzado tanto en el plano de lo social como en lo jurídico. Así, hoy por hoy la comprensión y aceptación de otras formas distintas de familia a la versión tradicional de padre-madre- 
hijos. Lo cual, nos lleva a la tercera pregunta cestá preparada la sociedad colombiana para la incorporación de familias homoparentales? La respuesta a cada una de estas preguntas requiere de una profunda reflexión en torno a las especificidades de cada cuestionamiento que gira en torno a los valores esenciales con los cuales una sociedad decide sus concepciones de vida buena.

La presencia de los movimientos sociales, es central al reconocimiento de cuestiones políticas y sociales, a la pluralidad de las formas de vida. Al estar en constante interacción con las instituciones del Estado en la búsqueda de objetivos concretos y específicos que tienen como propósito incorporar sus peticiones. Esta interacción resulta vital en el marco de una constitución garantista en derechos como lo es, la Constitución política colombiana de 1991. Dado que nuestro marco jurídico creó un sistema abierto de acceso para solicitar derechos es lógico que los ciudadanos se hicieran presente ante las instituciones del Estado para reclamarlos y hacerlos válidos. El logro de una sociedad civil libre y diferenciada ha sido considerada como uno de los rasgos característicos de las sociedades liberales (Sauca \& Wences, 2007) como garantía de que la participación ciudadana sea posible en la toma de las decisiones políticas.

La idea de la familia no deja de ser problemática, la cuestión como señala Diana Maffía, es abordarla en el estilo en que es imaginada. El feminismo clásico contribuyó en los estudios demográficos para hacer visibles los cambios sustanciales en las formas de convivencia pero sobre todo para mostrar que las unidades familiares no son homogéneas, ideales, también integran espacios de poder que a veces se expresan violentamente, pero "las familias ya no son tampoco -la posesión del patriarca- porque esos otros sujetos tienen derechos y deben poder hacerlos valer frente al Estado" (Maffía, 2012, p. 27). Los estudios producidos sobre la familia deben exponer de forma clara la autoridad del pater, los ciclos familiares y sus ritos de pasajes, las identidades sexuales y los intercambios con las distintas instituciones desde una perspectiva cultural y social.

La inclusión de las familias homoparentales es necesaria puesto que en la reconfiguración de los modos de participación de los colectivos el movimiento LGBTI se hace presente en la discusión en torno a la garantía de derechos. Es así que se apunta al reconocimiento de una especificidad cultural y al reclamo como sujetos plenos de derechos. Además, la violencia homofóbica, la discriminación en el mercado de trabajo, cuestiones relativas al derecho 
de adopción, de herencia y matrimonio pasan a ser tratados con inusitada frecuencia por diarios, revistas y programas de televisión" (Figari, 2010, p. 232). Es especialmente notable el esfuerzo teórico y político del feminismo, el cambio fundamental en las disciplinas sociales con respecto a las respuestas que debía proporcionar el Estado frente a las necesidades de las familias (Maffía, 2012). Sin embargo, para dar respuesta a los desafíos teóricos que implican el estudio de familias sexualmente diversas deben ampliarse los marcos teóricos y metodológicos de las ciencias sociales. Se deben incluir también los problemas jurídicos e incluso médicos que permitan ampliar el marco conceptual desde el cual la presencia de estas distintas formas de familia sea ampliamente reconocida.

\section{Jurisprudencia y movimientos sociales}

Tuestro objeto de estudio son las familias homoparentales en el Caribe colombiano tomando como punto coyuntural la sentencia

ـ C-811/07, la primera de su tipo en reconocer los derechos jurídicos de las parejas del mismo sexo. Hemos realizado una recopilación de las sentencias judiciales de la Corte Constitucional colombiana en materia de derechos para parejas LGBTI en Colombia, desde 1992 hasta 2015 para exponer el contexto previo que da paso a la consolidación del proceso social y su continuidad. En esta selección se han escogido aquellas sentencias que tengan relación con beneficios jurídicos como lo son: derecho a la seguridad social, pensión, porción conyugal y matrimonio.

El desarrollo de la jurisprudencia de la Corte Constitucional en torno a derechos de población LGBTI se puede dividir en dos periodos, el primero que va desde 1992 a 2006 y el segundo que va desde el 2007 hasta nuestros días el cual es el que nos compete analizar. En el primer periodo las resoluciones van en la dirección de la protección de los derechos individuales, la defensa de las libertades, la libre expresión y la autonomía, de forma especial, en las instituciones educativas y en espacios públicos. Desde el 2007 la evolución gira más hacia derechos colectivos. La sentencia C-811/07 es el primer reconocimiento de derechos de los compañeros permanentes del mismo sexo que viven en uniones de hecho. Es así como desde entonces se abre la posibilidad de discutir la violencia en (Colombia Diversa) trasfamiliar en las parejas del mismo sexo y que este también pueda ser digno de atención por las autoridades en los casos en los que se presente. La afiliación a la seguridad social (salud) y el derecho a reclamar la pensión de sobrevivientes en los casos en que el compañero permanente muera. Los temas en relación a beneficios 
jurídicos a las parejas del mismo sexo se resumen en la siguiente tabla:

Tabla 1. Sentencias referentes a conformación de familias de parejas del mismo sexo

\begin{tabular}{|c|c|c|}
\hline Tema & Sentencias & Total \\
\hline Adopción & $\begin{array}{l}\mathrm{T}-290 / 95 \\
\mathrm{C}-814 / 01 \\
\mathrm{C}-683 / 15\end{array}$ & 3 \\
\hline Seguridad social & $\begin{array}{c}\mathrm{T}-999 / 00 \\
\mathrm{~T}-618 / 00 \\
\text { SU-623/01 } \\
\mathrm{T}-1426 / 00 \\
\mathrm{~T}-349 / 06 \\
\mathrm{C}-811 / 07\end{array}$ & 6 \\
\hline Visita íntima en cárceles & $\begin{array}{l}\mathrm{T}-499 / 03 \\
\mathrm{~T}-624 / 05 \\
\mathrm{~T}-274 / 08\end{array}$ & 3 \\
\hline Matrimonio & $\begin{array}{c}\text { C-886/10 (Inhibitoria) } \\
\text { C-577/11 } \\
\text { SU- } 214 / 16\end{array}$ & 3 \\
\hline Uniones maritales de hecho & $\begin{array}{l}\mathrm{T}-725 / 04 \\
\mathrm{C}-798 / 08 \\
\mathrm{C}-029 / 09 \\
\mathrm{~T}-717 / 11\end{array}$ & 4 \\
\hline Herencia y pensión & $\begin{array}{c}\mathrm{C}-1043 / 06 \\
\mathrm{C}-336 / 08 \\
\mathrm{~T}-1241 / 08 \\
\mathrm{~T}-911 / 09 \\
\mathrm{~T}-051 / 10 \\
\mathrm{C}-283 / 11 \\
\mathrm{~T}-716 / 11 \\
\mathrm{C}-238 / 12 \\
\mathrm{~T}-357 / 13\end{array}$ & 9 \\
\hline
\end{tabular}

Fuente: Elaboración propia en base a resultados tomados del blog de la organización Colombia Diversa (Colombia Diversa, 2017)

Esta breve exposición del marco legal y jurídico que da vía libre a que se hable de familias lesbianas y gay en el país origina el punto de partida desde el cual la formalización de las uniones de hecho, así como las uniones solemnes y más adelante los matrimonios se van a formalizar y es posible la creación de índices cuantitativos. La utilización de esta expresión tiene sus ventajas y desventajas que son preciso aclarar en términos metodológicos pues cualquier intento de definir las familias se corren riesgos políticos e ideológicos. Teniendo en cuenta esto se sugiere que se definan "por la presencia de dos o más personas que comparten su predilección por el mismo sexo, orientación (por ejemplo, una 
pareja), o por la presencia de al menos un gay o lesbiana adulto y la crianza de un hijo" (Zapata, 2009: 18).

Los indicadores cuantitativos son un buen punto de partida para analizar el acceso diferenciado de los movimientos sociales a instancias de poder (Yon Leau, 1996). En nuestra investigación hemos consultado la base de datos de la Superintendencia de Notariado y Registro, con el fin de encontrar las uniones matrimoniales celebradas en la costa Caribe colombiana con el fin de analizar y proporcionar algunas respuestas sobre la conformación de familias de gays y lesbianas en la región, las dificultades, avances que permiten hablar de democratización de los espacios.

El análisis cuantitativo debe ser acompañado de metodologías alternas, para ello, hemos entrevistado a activistas del movimiento LGBTI en Cartagena, con el fin de obtener de primera mano cómo se posiciona el tema de la familia en la región. Es importante como el trabajo desde la gestión pública de algunos miembros y el trabajo comunitario de otros han permitido visibilizar el tema y cuestionar la idea de la familia monogámica heterosexual. Las entrevistas en profundidad son "herramientas de excavar", además de ser una metodología flexible y dinámica, ofrecen la oportunidad de investigar ampliamente sobre temas sensibles a personas que estuvieron involucradas con los hechos (Gray, Williamson, Karp, \& Dalphin, 2007).

Como se parte de que los integrantes de los colectivos narren sus historias sobre los hechos, existen una serie de dificultades inevitables en este tipo de metodologías de los cuales se debe hacer mención: uno es que muchos de ellos se rehúsan a hablar de manera que podríamos llamar "espontánea", se muestran con desconfianza ante el entrevistador y son parciales en sus respuestas. Segundo, puede darse una sobreexageración de sus emociones (Bosco; 2006) y por lo tanto, perder objetividad sobre los hechos. Para contrarrestar estos fenómenos la construcción de las narrativas como propuesta metodológica se hace de una forma exploratoria, totalmente flexible en la cual aún faltan datos que recolectar. Por narrativas entendemos como "la vinculación de creencias y prácticas asociadas a la identidad individual y colectiva con el lenguaje o conversación que las explica, así como la interacción contextual entre participantes de una relación, con su esfuerzo por explicarla e interpretarla" (Zapata, 2009:11) son una buena forma de acceder a aquello que es más difícil explicar a la hora de estudiar los fenómenos y hechos sociales: la forma como se establecen sus redes y los esfuerzos que realizan 
por lograr algo.

\section{Las familias diversas en Colombia}

n Colombia, la canalización de derechos de los grupos LGBTI se ha dado a lo largo de los últimos treinta años de historia por vía judicial y basada en discusiones jurídicas entre expertos del derecho (Albarracín, 2015). La movilización de grupos LGBTI ha estado limitada a las resoluciones judiciales y sigue habiendo un distanciamiento del Congreso. Si bien por vía judicial los grupos LGBTI han logrado la protección de sus derechos, existe aún el problema del trato en otras instituciones estatales a la hora de hacer cumplir con los mandatos judiciales y la falta de un programa de inclusión real de esta población, así como la sensibilización de funcionarios, políticos, fuerza pública y médicos en el tratamiento de lo que tiene que ver con población LGBTI.

Es importante poner de relieve los issues relativos a las razones por las que los ciudadanos y los grupos acuden a las cortes y no ante otras instituciones del Estado y de esta manera, explicar por qué no recurren a formas no litigiosas para trabajar en la resolución de sus conflictos. Las minorías empiezan a presentarse ante los jueces para hacer efectivas una serie de demandas, sean étnicas, religiosas, de género u otras. Es preciso señalar que no se puede seguir afianzando la idea de que la justicia social pueda ser definida en términos de reglas que hagan caso omiso a las diferencias (Carbonell, 2009). Para ello hace falta repasar los argumentos a favor y en contra de los derechos culturalmente diferenciados y aterrizar el debate cuando se hace referencia a derechos colectivos. Los grupos usan las cortes como un medio para proporcionar un contrapeso a la oposición que percibe de otras instancias de poder.

La sentencia del matrimonio de 2011 que permite que las parejas del mismo sexo puedan celebrar una "unión solemne" ante el notario o juez competente permitió separar la familia de la heterosexualidad. Si bien la legislación para modificar la ley de matrimonios no se llevó a cabo en el Congreso como ordenaba la sentencia, la parte resolutiva de la sentencia señalaba que en caso de no llevarse a cabo, los notarios del país podían celebrar las uniones. La cuestión del matrimonio aceleró el debate sobre la adopción y en la sentencia C-683/2015, el movimiento consiguió que las parejas del mismo sexo puedan recurrir a proceso de adopción. Finalmente, mediante sentencia SU- 214 de 2016 dio vía libre al matrimonio igualitario en Colombia.

Ahora bien, ni la jurisprudencia ni los proyectos de ley hasta ahora existentes 
han reflexionado sobre los siguientes interrogantes que quedan abiertos hacia el rumbo que tomará la lucha y las discusiones:

¿Qué pasará cuando una mujer lesbiana pida acceso a fertilización in vitro para ser portadora de un embrión con la donación de óvulo de su pareja? ¿Qué pasará cuando alguien que fue asignado como varón pida su cambio al género femenino, con modificación de documento y partida de nacimiento pero sin cirugía genital, y años después esa mujer transgénero solicite a su EPS una autorización para una operación de próstata? ¿Qué pasará cuando un varón trans que no ha modificado sus cuerpos, en pareja con otro varón decida gestar un hijo de la pareja y solicite como varón una licencia por maternidad? (Maffía, 2011:34).

Así mismo, no atiende a los contextos particulares donde las resoluciones se aplican. El matrimonio en la costa Caribe colombiana no es un factor determinante en la lucha del movimiento LGBTI de la región; de igual forma, los valores asociados al matrimonio y la conformación de familias diversas, se hace bajo los parámetros idiosincráticos de la región: la preferencia de la unión libre, la adopción informal de los menores y la crianza de los hijos en familias nucleares tradicionales, donde uno de los cónyuges es de orientación sexual diversa. Esto no quiere decir que la aprobación del matrimonio y la vía libre para adoptar no sean motivos para la celebración del movimiento de la región, pero hasta el momento no constituye un eje central de lucha política.

\section{El Caribe colombiano: retos y desafíos}

7 tlántico y Bolívar son los dos departamentos en la región donde ya existe un plan que permita implementar la política pública, seguidos

de las ciudades de Santa Marta, Soledad y Riohacha. En los demás departamentos de la región apenas empiezan a gestarse movimientos locales que impulsen planes de trabajo para la construcción de una política pública. Una de las organizaciones pioneras de la región ha sido Caribe Afirmativo quienes han estado trabajando desde hace unos años por la visibilización de los problemas y desafíos de la población LGBTI en la ciudad de Cartagena, impulsando poco a poco el trabajo en otras ciudades y municipios. La lucha se ha centrado sobre todo en una política de reconocimiento del uso y la presencia en el espacio público en la lucha contra la homofobia, la transfobia y la obtención de espacios en los concejos locales.

Según los integrantes de los movimientos de la ciudad, la política pública 
en especial de Cartagena -ciudad eje del movimiento en la región- "es un documento que recoge los problemas básicos de la población" (Duque, 2015). Sin embargo, la política pública no es una solución a los problemas sustanciales (Duarte, 2015) porque los recursos asignados por la administración son insuficientes y usados en las marchas de orgullo LGBTI, que a razón de muchos dirigentes no da cuenta del problema esencial y convierte la lucha política por el reconocimiento, en algo festivo. Es por ello, que el matrimonio no se encuentra en el documento de la política pública, esto se debe a una sencilla razón, consideran que hay luchas que tienen que ser primarias antes del matrimonio y por supuesto la adopción.

El tema de la conformación de la familia, no está en el horizonte cercano aunque hace parte de la agenda de los movimientos, por lo menos en la región, ahora, esto no quiere decir que en la práctica no existan familias diversas. En términos de los porcentajes según el informe de la Superintendencia de Registro y Notariado entre los meses de enero y mayo de 2015 se registraron en las notarías del país 125 uniones maritales entre personas del mismo sexo, el $64.80 \%$ de las uniones maritales son hombres y el 32.20\% son mujeres. Después de Bogotá el departamento de Antioquia es el que registra mayor número de uniones, en el caso del Caribe colombiano las cifras son pocas y aumenta de forma muy lenta, como se muestra en la tabla a continuación:

Tabla 2. Uniones maritales de personas del mismo sexo en la costa Caribe

\begin{tabular}{lcc}
\hline \multicolumn{1}{c}{ Departamento } & 2014 & 2015 \\
\hline Atlántico & 3 & 5 \\
\hline Bolívar & 0 & 0 \\
\hline Cesar & 0 & 0 \\
\hline Córdoba & 0 & 0 \\
\hline La Guajira & 0 & 0 \\
\hline Magdalena & 0 & 1 \\
\hline San Andrés Islas & 0 & 1 \\
\hline Sucre & 1 & 0 \\
\hline Total costa Caribe & 4 & 7 \\
\hline
\end{tabular}

Fuente: Superintendencia de Notariado y Registro

En base a nuestra investigación y los resultados cuantitativos señalados 
anteriormente, hemos determinado al menos cinco factores o el conjunto de ellos que explicaría la escasez de uniones de parejas del mismo sexo de manera legalizada y las razones por la cuales, las familias homoparentales responden a dinámicas propias de la región. Primero, el tema del matrimonio es un asunto de centro/periferia. En palabras de uno de los líderes del movimiento y que recoge la posición general, se señala que:

En Bogotá, se volvió un discurso político < hablemos de matrimonio igualitario $>$ porque ellos ya tenían política pública. Tenían avances significativos en participación política LGBTI, tenían ediles, tenían concejales, tenían alcalde, entonces... obvio a ellos no les interesaba hablar (de otros temas) ¿Cómo vamos a sensibilizar a las escuelas para que se creen centros de atención cuando las chicas trans lleguen? Ellos no tienen ese problema (Duque, 2015).

Lo que esto quiere decir es que los avances en materia de familia en la región no se pueden hacer en tanto no exista una línea de base que solucione problemas básicos de la población: derechos humanos, acceso a la educación, libertad de expresión, autoreconocimiento, salud sexual y mental, derecho al uso del espacio público. Puesto que sin estas garantías, el ejercicio de la ciudadanía se haya restringido por la no existencia de espacios de participación social y democrática.

Junto a este primer factor se une el segundo que es el desconocimiento: desconocimiento por parte de los jueces, de la sentencia y las leyes que protegen los derechos de la comunidad LGBTI. Si los jueces, que son los guardianes del sistema judicial, desconocen los procedimientos en estos casos es imposible llevarlos a cabo y por tanto, hay obstrucción a la justicia. Hasta antes de la aprobación del matrimonio igualitario, la refrendación ante la notaría donde se legaliza la unión, no era considerado como matrimonio, puesto que, no generaba los efectos jurídicos que si se ganan con el matrimonio, de ahí a que tampoco haya demasiadas uniones solemnes en la región.

El tercer factor es el aspecto idiosincrático de la población, el matrimonio no se ve como un objetivo a lograr aunque exista un ideal de ello. Tampoco concuerda con los proyectos de vida de los ciudadanos, quienes se sienten condicionados por el contexto al cual pertenecen y articulan la identidad de las personas. En este caso, los procedimientos que se dan a nivel jurídico no repercuten en la vida de las personas "no todas las personas LGBTI quieren 
casarse o tener hijos" (Duarte, 2015). El matrimonio es percibido como una cuestión de clase social y por ende, de estatus. Por ejemplo, una de los líderes señalaba como la diferencia de estratos sociales y así mismo, de locación geográfica condiciona la concepción sobre el matrimonio, se plantea:

Si vives en Bogotá, estrato seis y todo el tiempo has visto gente divina si te van a dar ganas de casarte. Si vives en Olaya idonde los persiguen! la gente no piensa en casarse sino en vivir los días, el sustento y el poquito de comida, o sea, eso no le va a pasar por la cabeza nunca y lo pueden escuchar y lo pueden saber, pero no hace parte de sus proyectos de vida. Porque sus proyectos de vida están destinados a otras cosas... Los contextos terminan dándole la identidad a las personas y terminan adecuándose a esa identidad (Duarte, 2015).

El matrimonio como un derecho que consolida la igualdad es percibido como un bien que no pueden usufructuar ni mucho menos como un beneficio esencial. En esta línea argumentativa, va la siguiente pregunta ¿Cómo una mujer trans en Cartagena estaría interesada en conformar familia mientras no puede costear los gastos que implican conseguir hormonas? El matrimonio no se constituye como un ideal a conseguir en tanto, los asuntos más básicos, como higiene y salud sexual, no se han resuelto en la ciudad.

El quinto y último factor es la baja tolerancia y el poco respeto a la diferencia de la ciudadanía en general. La cultura costeña, es heterosexista, eje de la estructura, el funcionamiento y las narrativas patriarcales. Pese a una creciente presencia de la población LGBTI en la sociedad, siguen sintiendo miedo a sentirse rechazado y percibir un ambiente hostil para realizarlo. Las agresiones a personas con diversidad sexual es constante, aunque existe cierto nivel de aceptación. Es preferible -según la opinión de muchos- que la vida de parejas sexualmente diversas se quede en el espacio de la vida íntima. Eso genera algunos conflictos en la vida cotidiana, la violencia intrafamiliar está presente en la vida de las parejas LGBTI. Debido a que no hay una sensibilización por parte de autoridades y funcionarios estas agresiones no son denunciadas, ni existen acciones jurídicas que permitan poner freno a situaciones de abuso por parte de la pareja, de una manera particular a las mujeres trans. La familia como espacio pluridimensional comporta distintas situaciones conflictivas que deben ser tenidas en cuenta por parte de los estudios de la familia y los que elaboran y diseñan las políticas. Dado que en Colombia se reconocen que las parejas conformadas por personas con orientación sexual diversa conforman 
y son familia, la inclusión de las problemáticas en estas son esenciales a la hora de armar los programas de protección a la familia.

Si bien hay pocas uniones formalizadas ante notario, son varios los casos de personas que se casan en la ciudad de Cartagena, provenientes del interior del país o incluso extranjeros, por la facilidad y el atractivo que ofrece la ciudad. Esto se debe a un referente de Cartagena como "el reino de lo posible". En Bogotá, por ejemplo, donde la presencia de grupos conservadores radicales está más presente, la celebración de este tipo de contratos es escasa. Es conocido el caso del juez, en un municipio del sur de Bolívar, quien ha realizado veinticinco matrimonios a través de la elaboración de los juicios y quien en garantía de la igualdad y en base a la sentencia ha celebrado varias uniones, siendo apodado como "el juez de los maricas". Este trabajo, sin duda ha tenido efectos al interior de la rama judicial porque para algunos sectores el trabajo de este juez no está bien visto.

Si es posible hablar de casos (por demás interesantes) de la conformación de familias lesbianas y gays e incluso de mujeres transexuales. La referencia de estos casos de los cuales tenemos referencias, resultan de la información obtenida en las entrevistas. A pesar de ello, el porcentaje de parejas del mismo sexo y de la existencia de familias homoparentales no es posible estimar porque los datos no existen, ni se han hecho las investigaciones propiamente dichas. Esta deficiencia de los datos genera la cuestión del problema metodológico si los censos y las investigaciones sociales tienen la sensibilidad y actualización como para recoger su variación en el tiempo y la geografía.

Existen así mismo innumerables casos de tíos homosexuales que crían a los sobrinos por el fallecimiento de los padres o en condiciones en las cuales, aquellos tienen mejores condiciones económicas para solventar los gastos de la crianza de los sobrinos. Un caso sobresaliente en una situación similar se da con la adopción en el municipio de Talaigua, Bolívar donde un joven homosexual adopta el hijo de una amiga, fruto de una violación y a quien la comisaria del municipio emite la orden de quitarle al niño y exponer al joven por no ser considerado "idóneo moralmente" para criar al niño.

Aún más paradójico es el caso de una mujer trans negra de un barrio popular en la ciudad de Cartagena, quien adopta y cría como madre soltera a unos niños. Este caso, se convierte en paradigmático, si bien tenemos en cuenta que la existencia de prejuicios en donde es enmarcada esta mujer tienen unas 
connotaciones peyorativas y discriminatorias: mujer-trans-negra-habitante de barrio popular. A pesar de ello y según los relatos de los activistas, esta mujer logra criar a sus hijos y después de muchos devenires, logra conseguir la aceptación de la comunidad donde vive. Estos casos están rodeados de cierta excentricidad y puede pasar mucho tiempo para llegar a ser aceptados por las comunidades en las que viven.

Todavía hace falta mucho para lograr una mejor comprensión de los mismos, una de las fallas con las que nos encontramos es que los profesores y la escuela en general como institución tanto privadas de clase media-media alta y escuelas públicas de clase media-baja/pobre no tienen conocimiento de la existencia de familias homoparentales en sus escuelas. Esta situación dificulta el trabajo de convivencia escolar para fomentar el respeto hacia la diversidad, en cierto sentido esta reserva es comprensible dado la fuerte cultura machista arraigada en la región ${ }^{1}$. Se requiere la construcción de un programa de inclusión social que involucre familia, escuela e instituciones del Estado donde la diversidad sea vista como un asunto que hay que incluir en la agenda de las ciudades.

Así como está en juego la visibilidad de las familias también se pone en juego los roles tradicionalmente asignados a la figura paterna y materna. De una forma especial en el Caribe el peso de la crianza recae sobre la madre, pero en el caso de las familias homoparentales, esta asignación se trastoca y rebasa la compresión básica asignada a los roles de hombres y mujeres en el proceso de crianza. El siguiente asunto de la agenda a resolver es el tema de la inclusión por parte de la sociedad en general pues uno de los obstáculos a los cuales se enfrentan las familias que no permitido el avance de la adopción es la falta de aceptación social. Mientras no se ve con problema que se casen o vivan en unión de hecho, el tema de adopción resulta más problemático. En el campo de las políticas públicas para erradicar la discriminación "es el cambio de este orden social y cultural discriminatorio, hacia uno que reconozca la plena igualdad" (Valdés, 2007). Es así como como consideramos que el enfoque apropiado desde el cual las políticas públicas deben construirse es desde una perspectiva de género que partan del reconocimiento de la condición de desigualdad y exclusión histórica que ha determinado roles, conductas e identidades aceptables para cada uno.

\section{Conclusiones}

1 Los casos de madres solteras son mucho más comunes y frecuentes y mayormente aceptados socialmente. 
$\mathrm{P}$ roponemos un eje donde el centro es la familia estudiada desde una triada que sería: enfoque de género-democracia-diversidad. Bajo este enfoque epistémico y metodológico, consideramos que el abordaje de la inclusión en las políticas públicas como una forma de construcción de democracia incluya un eje que es el de la familia diversa. El destacado trabajo por parte de los estudios de género ha permitido rescatar estas otras formas de conformar y ser familia, necesario para ampliar los márgenes de acción donde se piensan llevar las políticas públicas.

En el caso de las familias diversas ha sido fundamental la participación de los movimientos civiles que han llevado sus peticiones ante las instituciones del Estado para reclamar mayor igualdad. En Colombia, el actor institucional clave que ha permitido esta posibilidad ha sido la Corte Constitucional colombiana, como un ente garantista en la protección de los derechos de la comunidad LGBTI; sin dejar de lado, la presencia constante de los movimientos en la escena política. Se ha logrado el reconocimiento jurídico de la existencia de las familias diversas, derechos patrimoniales y de afiliación a la seguridad y de forma más reciente la posibilidad de adoptar por parte de parejas del mismo sexo y el matrimonio igualitario. Esto sin duda conduciría a plantear otros debates, que serán determinados por el avance de las políticas.

Faltan investigaciones por hacer así como una ampliación de los conocimientos sobre las regiones en particular. Es necesario la construcción de censos y bases de datos que permitan tener un número de familias homoparentales, su ubicación para establecer planes de inclusión social que favorezcan la democratización de los espacios. Creemos que este trabajo es un aporte a esos estudios, sin embargo, es importante hacerlos desde una perspectiva comparada con otras regiones y de esta manera, rescatar aquellas ideas que han dado efecto para obtener un mayor reconocimiento.

\section{REFERENCIAS BIBLIOGRÁFICAS}

Cicerchia, R. (2012). Pobreza estructural, políticas públicas y performance: la encrucijada de las formas familiares en América Latina. I Memorias del I Seminario Internacional y II Nacional de Familias Contemporáneas y Políticas Públicas, (págs. 2-26). Cartagena.

Colombia Diversa (s.f.). Colombia-diversa. Recuperado el 8 de unio de 2017, de http://www. colombia-diversa.org/p/sentencias.html

Figari, C. (2010). El movimiento LGBT en América Latina: institucionalizaciones oblicuas. En A. 
Massetti, E. Villanueva, \& M. Gómez, Movilizaciones, protestas e identidades colectivas en la Argentina del bicentenario (págs. 225-240). Buenos Aires: Nueva Trilce.

Albarracín, M. (25 de 09 de 2015). La despenalización de los actos homosexuales en Colombia: un cambio legal desde arriba. Obtenido de http://www.malbarracin.com/: https://docs.google.com/ document/pub?id=1YgtP74Og2cyRDa-BIePyH4HjlZh8FK7V_FudKfLSrEk

Maffía, D. (2012). Familias diversas, iguales derechos. Avances legales y demandas pendientes. I Seminario Internacional y II Nacional de Familias Contemporáneas y Políticas Públicas (págs. 26-35). Cartagena: Ed. Universidad de Cartagena.

Yon Leau, C. (1996). Hacia el género desde las cifras: cuando se cuantifican las diferencias. En P. Ruiz-Bravo, Detrás de la puerta: hombres y mujeres en el Perú de hoy (págs. pp.221-264). Lima: Pontificia Universidad Católica del Perú.

Valdés, T. (2007). Igualdad de oportunidades y equidad de género. Aportes y desafíos para las políticas públicas. Santiago de Chile: Friedrich Ebert Stinftung.

Sauca, J. M. \& Wences, M. I. (2007). Lecturas de la sociedad civil. Un mapa contemporáneo de sus teorías. Madrid: Trotta.

Cohen, J. \& Arato, A. (2000). Sociedad civil y teoría política. México: FCE.

Whitehead, L. (2011). Democratización. Teoría y experiencia. México: FCE.

Rosanvallon, P. (2006). La contrademocracia. La política en la era de la desconfianza. Buenos Aires: Manantial.

Carbonell, M. (2009). Neoconstitucionalismo(s). Madrid: Editorial Trotta.

Duque, A. (27 de noviembre de 2015). Movimientos LGBTI en el Caribe. (L. P. López Bajo, entrevistador).

Duarte, T. (28 de noviembre de 2015). Movimiento LGBTI en el Caribe. (L. P. López Bajo, entrevistador).

Zapata, B. (2009). Homoparentalidad en Colombia. Provocación de la diversidad familiar. Bogotá: Universidad Nacional de Colombia. 